12,13

\title{
Влияние упругих напряжений на формирование осевых гетеропереходов в трехкомпонентных нитевидных нанокристаллах $A^{\text {III }} \mathrm{B}^{\mathrm{V}}$
}

\author{
(C) А.А. Корякин ${ }^{1,2}$, Е.Д. Лещенко ${ }^{3,2}$, В.Г. Дубровский \\ ${ }^{1}$ Санкт-Петербургский национальный исследовательский академический университет РАН, \\ Санкт-Петербург, Россия \\ ${ }^{2}$ Санкт-Петербургский национальный исследовательский университет \\ информационных технологий, механики и оптики, \\ Санкт-Петербург, Россия \\ ${ }^{3}$ Solid State Physics and NanoLund, Lund University, Box 118, \\ S-22100 Lund, Sweden \\ E-mail: koryakinaa@spbau.ru
}

Поступила в Редакцию 16 июля 2019 г.

В окончательной редакции 16 июля 2019 г.

Принята к публикации 25 июля 2019 г.

\begin{abstract}
Проведено теоретическое исследование влияния упругих напряжений на формирование осевых гетеропереходов в трехкомпонентных нитевидных нанокристаллах $\mathrm{A}^{\mathrm{III}} \mathrm{B}^{\mathrm{V}}$. Построены профили состава осевого гетероперехода InAs/GaAs в самокаталитических нитевидных нанокристаллах $\mathrm{Ga}_{x} \mathrm{In}_{1-x} \mathrm{As}$. Показано, что ширина гетероперехода InAs/GaAs составляет десятки монослоев и возрастает с увеличением радиуса нитевидного нанокристалла из-за упругих напряжений. Релаксация упругих напряжений на боковых поверхностях нитевидных нанокристаллов при типичной температуре роста (около $450^{\circ} \mathrm{C}$ ) и радиусе нитевидных нанокристаллов больше $5 \mathrm{~nm}$ не приводит к возникновению области несмешиваемости в системе $\mathrm{Ga}_{x} \operatorname{In}_{1-x}$ As.
\end{abstract}

Ключевые слова: нитевидные нанокристаллы, полупроводники III-V, гетероструктуры, эпитаксия.

DOI: $10.21883 /$ FTT.2019.12.48574.31ks

\section{1. Введение}

Нитевидные нанокристаллы (ННК) трехкомпонентных полупроводников $\mathrm{A}^{\mathrm{III}} \mathrm{B}^{\mathrm{V}}$ с осевыми гетеропереходами являются перспективными наноматериалами для создания на их основе новых оптоэлектронных приборов. В частности, с использованием ННК $\mathrm{A}^{\mathrm{III}} \mathrm{B}^{\mathrm{V}}$ был создан источник одиночных фотонов [1] и фотодетектор высокого пространственного разрешения [2]. В настоящее время широкое распространение получили эпитаксиальные методы роста НHК $\mathrm{A}^{\mathrm{III}} \mathrm{B}^{\mathrm{V}}$ по механизму пар-жидкость-кристалл (ПЖК). В этом случае рост ННК происходит на подложках, активированных каплями катализатора. В качестве катализатора часто используют золото или серебро [3]. Также возможен самокаталитический рост НHК $\mathrm{A}^{\mathrm{III}} \mathrm{B}^{\mathrm{V}}$, когда катализатором является элемент группы III (например, $\mathrm{Ga}$ [4]). В работах [5,6] была продемонстрирована возможность формирования осевых гетеропереходов в трехкомпонентных ННК $\mathrm{A}^{\mathrm{III}} \mathrm{B}^{\mathrm{V}}$ по ПЖК механизму. Величина упругих напряжений при когерентном сопряжении двух полупроводников, формирующих осевой гетеропереход, была определена в работах $[7,8]$. Обнаружено, что на расстояниях от гетерограницы, много меньших радиуса ННК, плотность упругой энергии близка к плотности упругой энергии, возникающей при когерентном росте тонкой пленки. С увеличением расстояния от гетерограницы плотность упругой энергии быстро убывает и на расстояниях порядка двух радиусов ННК приближенно равна нулю. Это связано с эффективной релаксацией упругих напряжений на боковых поверхностях ННК. Хорошо известно, что упругие напряжения могут существенно влиять на термодинамику наносистем $[9,10]$. Однако, существующие модели формирования осевых гетеропереходов в трехкомпонентных ННК $\mathrm{A}^{\mathrm{III}} \mathrm{B}^{\mathrm{V}}$ [4,11-13] построены без их учета. Пренебрежение упругими напряжениями допустимо, если рассматривать системы материалов, в которых максимально возможное рассогласование решеток мало (например $\mathrm{Al}_{x} \mathrm{Ga}_{1-x} \mathrm{As}$ ), или если осевой гетеропереход характеризуется малым изменением состава. В то же время, для приборных приложений часто представляет интерес создание максимально узких гетеропереходов между полупроводниками с большим рассогласованием решеток $[1,2]$. Цель настоящей работы состояла в оценке изменения ширины осевого гетероперехода в трехкомпонентных ННК $\mathrm{A}^{\mathrm{III}} \mathrm{B}^{\mathrm{V}}$, сформированных методом ПЖК, из-за влияния упругих напряжений. Расчет профиля состава осевого гетероперехода выполнен в рамках модифицированной модели [4]. 


\section{2. Теоретическая модель}

Рассмотрим формирование ННК $A_{x} B_{1-x} D$, где $A$ и $B$ - элементы группы III, $D$ - элемент группы V, c oceвым гетеропереходом $B D / A_{x} B_{1-x} D$, созданным путем переключения потока $B$ на поток $A$. Предположим, что ННК имеет форму цилиндра с радиусом $R$, на вершине которого находится сферическая капля катализатора с контактным углом $\beta$. Монослои сегмента ННК $A_{x} B_{1-x} D$ нумеруются, начиная с $i=1$. Состав капли катализатора при формировании $i$-го монослоя задается переменными $y_{i}$ и $c_{\text {tot }, i}: y_{i}=c_{A, i} /\left(c_{A, i}+c_{B, i}\right)$ и $c_{\text {tot }, i}=c_{A, i}+c_{B, i}$, где $c_{A, i}$ и $c_{B, i}$ - концентрация элементов $A$ и $B$ в капле, причем $c_{A, i}+c_{B, i}+c_{D, i}=1$, где $c_{D, i}-$ концентрация элемента $D$, если рост происходит по самокаталитическому механизму. Состав $i$-го монослоя $x_{i}$ является функцией переменных $y_{i}$ и $c_{\text {tot }, i}[4,11,14]$, а также является, в общем случае, функцией состава предыдущих монослоев $x_{i-1}, x_{i-2}$ и т.д.

$$
x_{i}=x_{i}\left(y_{i}, c_{\text {tot }, i}, x_{i-1}, x_{i-2}, \ldots\right) .
$$

Следуя работе [4], определим зависимость (1) во время роста ННК, используя равенство химических потенциалов для частиц в монослое и капле катализатора:

$$
\mu_{A, i}^{l}+\mu_{D, i}^{l}-\mu_{A D, i}^{s}=0 \text { и } \mu_{B, i}^{l}+\mu_{D, i}^{l}-\mu_{B D, i}^{s}=0,
$$

где $\mu_{A, i}^{l}, \mu_{B, i}^{l}$ и $\mu_{D, i}^{l}-$ химические потенциалы частиц $A, B$ и $D$ в капле катализатора; $\mu_{A D, i}^{s}$ и $\mu_{B D, i}^{s}-$ химические потенциалы пар $A D$ и $B D$ в растущем монослое. Таким образом, мы предполагаем, что зависимость состава монослоя от состава капли катализатора и состава предыдущих монослоев во время роста ННК совпадает с данной зависимостью в случае термодинамического равновесия между монослоем и каплей. В работе [4] в рамках данного термодинамического подхода были построены профили состава осевого гетероперехода в самокаталитических НHК $\mathrm{Al}_{x} \mathrm{Ga}_{1-x} \mathrm{As}$. Важно отметить, что результаты моделирования находятся в хорошем соответствии с экспериментальными данными [4].

Химические потенциалы частиц в капле катализатора и в монослое находятся в рамках модели регулярного раствора [11]. Химические потенциалы $\mu_{A, i}^{l}, \mu_{B, i}^{l}$ и $\mu_{D, i}^{l}$ определяются следующими формулами

$$
\begin{aligned}
\mu_{A, i}^{l}={ }^{o} \mu_{A}^{l} & +k_{\mathrm{B}} T \ln c_{A, i}+\omega_{A B} c_{B, i}^{2}+\omega_{A D} c_{D, i}^{2} \\
& +\left(\omega_{A B}+\omega_{A D}-\omega_{B D}\right) c_{B, i} c_{D, i}, \\
\mu_{B, i}^{l}={ }^{o} \mu_{B}^{l} & +k_{\mathrm{B}} T \ln c_{B, i}+\omega_{A B} c_{A, i}^{2}+\omega_{B D} c_{D, i}^{2} \\
& +\left(\omega_{A B}+\omega_{B D}-\omega_{A D}\right) c_{A, i} c_{D, i}, \\
\mu_{D, i}^{l}={ }^{o} \mu_{D}^{l} & +k_{\mathrm{B}} T \ln c_{D, i}+\omega_{A D} c_{A, i}^{2}+\omega_{B D} c_{B, i}^{2} \\
& +\left(\omega_{A D}+\omega_{B D}-\omega_{A B}\right) c_{A, i} c_{B, i},
\end{aligned}
$$

где ${ }^{o} \mu_{A}^{l},{ }^{o} \mu_{B}^{l}$ и ${ }^{o} \mu_{D}^{l}-$ химические потенциалы чистых элементов в жидком состоянии, $\omega_{A B}, \omega_{A D}$ и $\omega_{B D}-$ парные коэффициенты взаимодействия в жидкости, $k_{\mathrm{B}}$ постоянная Больцмана, $T$ - температура. При решении системы (2) в формулах (3) производится замена: $c_{A, i}=y_{i} c_{\mathrm{tot}, i}, c_{B, i}=\left(1-y_{i}\right) c_{\mathrm{tot}, i}$ и $c_{D, i}=1-c_{\mathrm{tot}, i}$. Химические потенциалы пар $A D$ и $B D$ в монослое $i$ содержат вклады от упругой энергии образования монослоя, $\mu_{A D, i}^{\text {elas }}$ и $\mu_{B D, i}^{\text {elas }}$, и определяются по формулам [10]:

$$
\begin{aligned}
& \mu_{A D, i}^{s}={ }^{o} \mu_{A D}^{s}+k_{\mathrm{B}} T \ln x_{i}+w\left(1-x_{i}\right)^{2}+\mu_{A D, i}^{\text {elas }}, \\
& \mu_{B D, i}^{s}={ }^{o} \mu_{B D}^{s}+k_{\mathrm{B}} T \ln \left(1-x_{i}\right)+w x_{i}^{2}+\mu_{B D, i}^{\text {elas }},
\end{aligned}
$$

где ${ }^{o} \mu_{A D}^{s}$ и ${ }^{o} \mu_{B D}^{s}-$ химические потенциалы чистых coединений $A D$ и $B D, w$ - коэффициент взаимодействия пар $A D$ и $B D$ в твердом теле. Вклады $\mu_{A D, i}^{\text {elas }}$ и $\mu_{B D, i}^{\text {elas }}$ находятся по следующим формулам: $\mu_{A D, i}^{\text {elas }}=\partial G_{i}^{\text {elas }} / \partial N_{A D, i}$, $\mu_{B D, i}^{\text {elas }}=\partial G_{i}^{\text {elas }} / \partial N_{B D, i}, \quad$ где $G_{i}^{\text {elas }}-$ избыток энергии Гиббса образования монослоя, связанный с упругими напряжениями, $N_{A D, i}$ и $N_{B D, i}$ - число пар $A D$ и $B D$ в монослое. Избыток энергии Гиббса $G_{i}^{\text {elas }}=W_{i}^{\text {elas }} N_{M L}$, где $W_{i}^{\text {elas }}-$ средняя упругая энергия, приходящаяся на одну пару $\mathrm{A}^{\mathrm{III}} \mathrm{B}^{\mathrm{V}}$ в $i$-м монослое, $N_{M L}-$ число пар $\mathrm{A}^{\mathrm{III}} \mathrm{B}^{\mathrm{V}}$ в монослое. Тогда

$$
\begin{gathered}
\mu_{A D, i}^{\text {elas }}=W_{i}^{\text {elas }}+\left(1-x_{i}\right) \partial W_{i}^{\text {elas }} / \partial x_{i}, \\
\mu_{B D, i}^{\text {elas }}=W_{i}^{\text {elas }}-x_{i} \partial W_{i}^{\text {elas }} / \partial x_{i} .
\end{gathered}
$$

Для самосогласованного определения состава растущего монослоя $x_{i}$ необходимо учесть уравнение материального баланса для вещества в капле катализатора. С целью получения простейших оценок ширины гетероперехода сделаем следующие предположения $[4,11]$. Во-первых, концентрации элементов $A$ и $D$ в капле много меньше концентрации элемента $B\left(c_{A, i} \ll c_{B, i}\right.$ и $c_{D, i} \ll c_{B, i}$ для любого $\left.i\right)$. Тогда объем капли во время формирования гетероперехода меняется слабо и определяется числом атомов элемента $B$ до переключения потоков. Можно показать, что уменьшение числа атомов элемента $B$ после переключения потоков не приводит к существенному изменению общего числа атомов в капле $N$ за время формирования гетероперехода, поэтому мы полагаем $N \approx$ const. Во-вторых, скорость роста ННК определяется потоком группы V, величина которого не меняется при формировании гетероперехода. В-третьих, пренебрежем изменением радиуса ННК $R$, следовательно, с учетом постоянства $N$ находим, что $\beta \approx$ const. В рамках сделанных предположений достаточно рассмотреть уравнение материального баланса только для атомов элемента $A$ в капле катализатора. Изменение числа атомов элемента $A$ в капле $N_{i}$ за время $\Delta t_{i}$ формирования $i$-го монослоя определяется по формуле

$$
N_{i}-N_{i-1}=\left(J-N_{M L} V x_{i}\right) \Delta t_{i}
$$

где $J$ - поток атомов элемента $A$ в каплю $\left(\mathrm{s}^{-1}\right)$, $V \approx$ const - скорость роста ННК (в $\left.\mathrm{ML} \mathrm{s}^{-1}\right)$. С учетом того, что $y_{i} \approx N_{i} / N$ и $V \Delta t_{i} \approx 1 \mathrm{ML}$ для любого $i$, а 
также при пренебрежении разностью высот монослоев, уравнение материального баланса (6) записывается в виде:

$$
\begin{gathered}
y_{i}\left(c_{\mathrm{tot}, i}, x_{i}, x_{i-1}, \ldots\right)-y_{i-1}\left(c_{\mathrm{tot}, i-1}, x_{i-1}, x_{i-2}, \ldots\right) \\
\approx p\left(q-x_{i}\right),
\end{gathered}
$$

где $p=N_{M L} / N$ и $q=J / N_{M L} V$ - параметры модели, $y_{i}=y_{i}\left(c_{\text {tot }, i}, x_{i}, x_{i-1}, \ldots\right)-$ функция обратная функции (1).

На каждом шаге моделирования роста ННК вначале численно находится упругая энергия $W_{i}^{\text {elas }}$ как функция состава монослоя $x_{i}$ с учетом заданных составов предыдущих монослоев $x_{i-1}, x_{i-2}, \ldots$ З Затем из решения системы уравнений (2) с использованием формул $(3)-(5)$ находится функция $y_{i}\left(c_{\mathrm{tot}, i}, x_{i}, x_{i-1}, \ldots\right)$, где $x_{i-1}, x_{i-2}, \ldots$ выступают в качестве параметров, значение $c_{\text {tot, } i}$ близко к $1[4,11,14]$ и его изменением пренебрегаем. Наконец, состав $i$-го монослоя определяется из решения уравнения (7) относительно $x_{i}$. Упругая энергия $W_{i}^{\text {elas }}\left(x_{i}\right)$ вычисляется с использованием метода конечных элементов (МКЭ) [15]. При реализации МКЭ предполагается, что боковые поверхности ННК и поверхность верхнего монослоя находятся в ненагруженном состоянии. Длина сегмента $B D$ выбирается такой, чтобы ее величина не влияла на результаты расчетов. С помощью МКЭ вычисляется суммарная упругая энергия системы $W_{\mathrm{tot}, i}^{\mathrm{elas}}\left(x_{i}\right)$, состоящей из сегмента $B D$ и $i$ монослоев сегмента $A_{x} B_{1-x} D$. Упругая энергия образования $i$-го монослоя находится по формуле:

$$
W_{i}^{\text {elas }}\left(x_{i}\right)=W_{\text {tot }, i}^{\text {elas }}\left(x_{i}\right) / N_{M L}-\sum_{k=0}^{i-1} W_{k}^{\text {elas }}\left(x_{k}\right),
$$

где $W_{0}^{\text {elas }}\left(x_{0}\right)=0$.

\section{3. Результаты и обсуждение}

В качестве примера материальной системы рассмотрим формирование осевого гетероперехода InAs/GaAs в самокаталитических НHК $\mathrm{Ga}_{x} \mathrm{In}_{1-x}$ As co структурой сфалерита, растущих в направлении [111]. Результаты моделирования, проведенного при характерной температуре роста НHK $\mathrm{Ga}_{x} \mathrm{In}_{1-x}$ As $T=450^{\circ} \mathrm{C}$ [11], представлены на рис. 1-3. Коэффициенты взаимодействия в капле катализатора и в твердом теле были определены на основе справочных данных [16]: $\omega_{\mathrm{GaIn}}=4450.0+1.19185 T \mathrm{~J} \mathrm{~mol}^{-1}, \quad \omega_{\mathrm{GaAs}}=-25503.6$ $-4.3109 T \mathrm{~J} \mathrm{~mol}^{-1}, \omega_{\mathrm{InAs}}=-15851.0-11.27053 T \mathrm{~J} \mathrm{~mol}^{-1}$, $w=19698.8-7.516937 \mathrm{~J} \mathrm{~mol}^{-1}$. Разности химических потенциалов ${ }^{o} \mu_{\mathrm{Ga}}^{l}+{ }^{o} \mu_{\mathrm{As}}^{l}-{ }^{o} \mu_{\mathrm{GaAs}}^{s}$ и ${ }^{o} \mu_{\mathrm{In}}^{l}+{ }^{o} \mu_{\mathrm{As}}^{l}-{ }^{o} \mu_{\mathrm{InAs}}^{s}$, найдены как функции температуры также с использованием справочных данных [16]. Значение параметров модели $p$ и $q$ для системы $\mathrm{Ga}_{x} \mathrm{In}_{1-x} \mathrm{As}$ были оценены в работе [11]: $p=0.0015$ и $q=1$. Для определения упругих констант и постоянной



Рис. 1. Зависимости упругой энергии, приходящейся на одну пару $\mathrm{A}^{\mathrm{III}} \mathrm{B}^{\mathrm{V}}$ в $i$-м монослое сегмента $\mathrm{Ga}_{x} \mathrm{In}_{1-x} \mathrm{As}$, от состава монослоя. Зависимости построены для монослоев с номером $i=1$ и радиусом ННК $R=30$ (1), 20 (2), 10 (3) и $5 \mathrm{~nm}(4)$. В случае $R=30 \mathrm{~nm}$ построены также зависимости для монослоев с номерами $i$, равными 11, 21, 31 и 41 (стрелкой обозначено направление сдвига минимума зависимости при увеличении $i$ ). Цифрой 5 обозначена зависимость упругой энергии образования тонкой пленки $\mathrm{Ga}_{x} \operatorname{In}_{1-x} \mathrm{As}$ на подложке InAs.

решетки $\mathrm{Ga}_{x} \operatorname{In}_{1-x} \mathrm{As}$ использовались значения материальных констант GaAs и InAs и предполагалось выполнение правила Вегарда. Постоянная решетки $\mathrm{Ga}_{x} \mathrm{In}_{1-x} \mathrm{As}$, используемая в расчете, $a=$ $=(0.60583-0.0405 x) \mathrm{nm}$ [17]. Согласно справочным данным [17], коэффициенты упругости GaAs равны: $c_{11}=119 \mathrm{GPa}, c_{12}=53.4 \mathrm{GPa}, c_{44}=59.6 \mathrm{GPa}$, а коэффициенты упругости InAs равны: $c_{11}=83.4 \mathrm{GPa}$, $c_{12}=45.4 \mathrm{GPa}, c_{44}=39.5 \mathrm{GPa}$. Важно отметить, что данные упругие константы соответствуют объемному материалу. Упругие же свойства отдельных монослоев могут существенно отличаться, причем их модули упругости могут быть как меньше, так и больше, чем у объемного материала [18]. Однако мы полагаем, что используемые значения упругих констант являются хорошим приближением для упругих констант монослоя, находящегося на поверхности объемного материала.

Из зависимостей, изображенных на рис. 1, видно, что при больших рассогласованиях решеток упругая энергия $W_{i}^{\text {elas }}\left(x_{i}\right)$ может достигать значения $200 \mathrm{meV}$ на одну пару $\mathrm{A}^{\mathrm{III}} \mathrm{B}^{\mathrm{V}}$. Из расчета МКЭ также следует, что плотность упругой энергии растущего монослоя существенно уменьшается только вблизи боковых поверхностей ННК. Например, на расстоянии $1-5 \mathrm{~nm}$ от боковых поверхностей плотность упругой энергии в несколько раз ниже, чем в центре ННК. На рис. 2 представлены зависимости состава монослоя $x_{i}$ от состава капли катализатора $y_{i}$, рассчитанные для нескольких монослоев сегмента $\mathrm{Ga}_{x} \operatorname{In}_{1-x}$ As. Для сравнения также построена зависимость состава монослоя от состава 


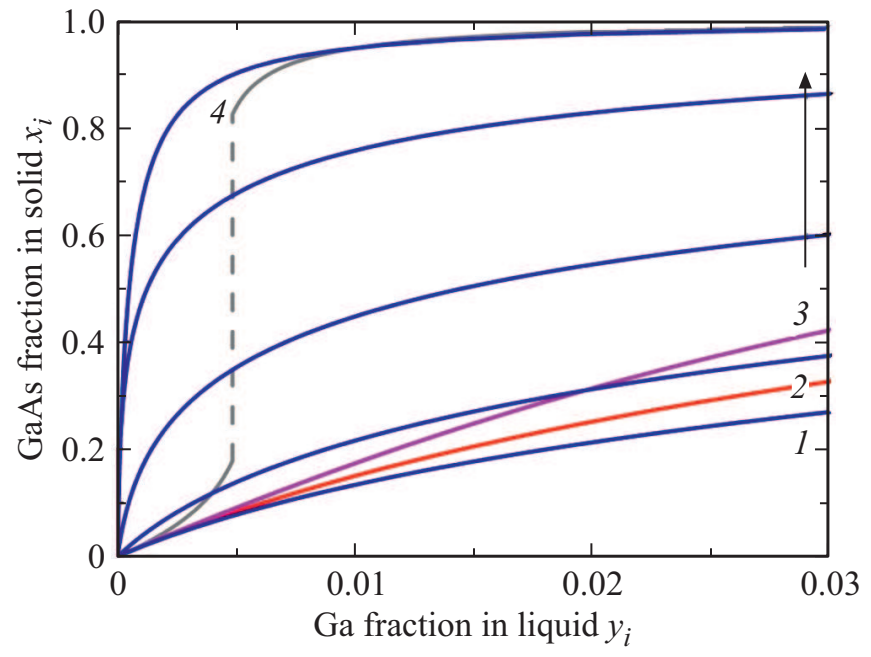

Pис. 2. 2. Зависимости состава монослоя от состава капли катализатора. Зависимости построены для монослоев с номером $i=1$ и радиусом ННК $R=30(1), 10$ (2) и $5 \mathrm{~nm}(3)$. Зависимости для ННК радиусом $R=20$ и $30 \mathrm{~nm}$ неразличимы при $i=1$. В случае $R=30 \mathrm{~nm}$ построены также зависимости для монослоев с номероами $i$, равными 11, 21, 31 и 41 (стрелкой обозначено направление возрастания номера $i$ ). Цифрой 4 обозначена зависимость состава монослоя от состава капли катализатора, построенная без учета упругих напряжений [11].

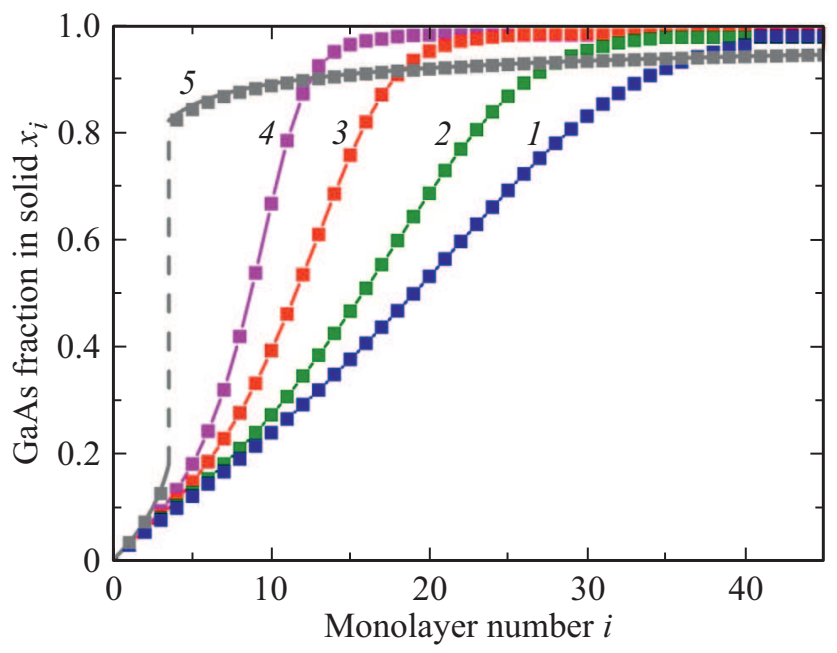

Рис. 3. Профили состава осевой гетероструктуры InAs/GaAs в ННК радиусом $R=30(1), 20(2), 10$ (3) и $5 \mathrm{~nm}(4)$. Также построен профиль состава (5) без учета упругих напряжений [11].

капли без учета упругих напряжений $x_{i}^{0}\left(y_{i}^{0}\right)$. График зависимости $x_{i}^{0}\left(y_{i}^{0}\right)$, построенный в настоящей работе, совпадает с графиком, рассчитанным в работе [11]. Из сравнения графиков на рис. 2 следует, что учет упругих напряжений ведет к кардинальному изменению диаграммы состава. Совпадение полученных зависимостей с $x_{i}^{0}\left(y_{i}^{0}\right)$ наблюдается только для первых монослоев сегмента $\mathrm{Ga}_{x} \operatorname{In}_{1-x}$ As при $x_{i}$, стремящемся к 0 , и для монослоев сегмента $\mathrm{Ga}_{x} \operatorname{In}_{1-x}$ As при $x_{i}$, стремящемся к 1, если составы нескольких предыдущих монослоев также близки к 1. В этих случаях упругими напряжениями можно пренебречь. Важно отметить, что учет упругих напряжений приводит к отсутствию области несмешиваемости $x_{i}=0.18-0.82[11]$ на диаграмме состава монослоя (рис. 2). Этот факт объясняется возникновением больших значений упругих напряжений при формировании двух последовательных монослоев, сильно рассогласованных по параметру решетки. Используя уравнение $\partial \mu_{A D, i}^{s} / \partial x_{i}=\partial \mu_{B D, i}^{s} / \partial x_{i}=0$ [10], находим, что область несмешиваемости не существует при температуре больше $\sim 130^{\circ} \mathrm{C}$, если радиус ННК больше $5 \mathrm{~nm}$. Отметим также, что рассчитанные значения переменных, задающих состав капли, меняются слабо и равны $c_{\text {tot }, i} \sim 0.98$ и $y_{i} \sim 0.01$ для любого $i$. Следовательно, условие $c_{A, i} \ll c_{B, i}$ и $c_{D, i} \ll c_{B, i}$ выполнено при любом $i$. Профили состава осевого гетероперехода построены на рис. 3. Общей особенностью полученных зависимостей также является отсутствие области несмешиваемости при учете упругих напряжений. Характерная ширина гетероперехода составляет около 15-40 монослоев и возрастает с увеличением радиуса ННК. Моделирование роста ННК различного радиуса произведено при одних и тех же значениях параметров $p$ и $q$. Это позволило оценить изменение ширины осевого гетероперехода, вызванное влиянием упругих напряжений, без учета других эффектов. Например, известно, что „эффект резервуара“ также приводит к возрастающей зависимости ширины гетероперехода от радиуса ННК [19].

\section{4. Заключение}

Таким образом, изучено влияние упругих напряжений на формирование осевых гетеропереходов в трехкомпонентных ННК $\mathrm{A}^{\mathrm{III}} \mathrm{B}^{\mathrm{V}}$. В качестве примера материальной системы рассмотрена система $\mathrm{Ga}_{x} \mathrm{In}_{1-x}$ As. Построены профили состава осевого гетероперехода InAs/GaAs в самокаталитических НHK $\mathrm{Ga}_{x} \mathrm{In}_{1-x}$ As. Обнаружено, что ширина осевого гетероперехода InAs/GaAs составляет 15-40 монослоев и возрастает с увеличением радиуса ННК. Также показано, что при типичных значениях температуры роста и радиуса ННК релаксации упругих напряжений на боковых поверхностях ННК недостаточно для возникновения области несмешиваемости в системe $\mathrm{Ga}_{x} \mathrm{In}_{1-x} \mathrm{As}$. Представленные результаты получены в рамках термодинамического подхода к определению зависимости состава монослоя от состава капли катализатора и состава предыдущих монослоев. Необходимо отметить, что кинетика поступления вещества к растущему монослою также может существенно влиять на его состав. Поэтому дальнейшее изучение кинетических процессов необходимо для более полного понимания механизмов роста ННК $\mathrm{A}^{\mathrm{III}} \mathrm{B}^{\mathrm{V}}$. 


\section{Финансирование работы}

Работа выполнена при финансовой поддержке Российского фонда фундаментальных исследований (проект № 18-32-00559).

\section{Конфликт интересов}

Авторы заявляют, что у них нет конфликта интересов.

\section{Список литературы}

[1] S. Deshpande, J. Heo, A. Das, P. Bhattacharya. Nature Commun. 4, 1675 (2013).

[2] M.P. Kouwen, M.H.M. Weert, M.E. Reimer, N. Akopian, U. Perinetti, R.E. Algra, E.P.A.M. Bakkers, L.P. Kouwenhoven, V. Zwiller. Appl. Phys. Lett. 97, 113108 (2010).

[3] E.K. Mårtensson, A.M. Whiticar, M. de la Mata, R.R. Zamani, J. Johansson, J. Nygård, K.A. Dick, J. Bolinsson. Cryst. Growth Des. 18, 6702 (2018).

[4] G. Priante, F. Glas, G. Patriarche, K. Pantzas, F. Oehler, J.-C. Harmand. Nano Lett. 16, 1917 (2016).

[5] K. Hiruma, H. Murakoshi, M. Yazawa, T. Katsuyama, J. Cryst. Growth 163, 226 (1996).

[6] L. Samuelson, C. Thelander, M.T. Björk, M. Borgström, K. Deppert, K.A. Dick, A.E. Hansen, T. Mårtensson, N. Panev, A.I. Persson, W. Seifert, N. Sköld, M.W. Larsson, L.R. Wallenberg. Physica E 25, 313 (2004).

[7] E. Ertekin, P.A. Greaney, D.C. Chrzan, T.D. Sands. J. Appl. Phys. 97, 114325 (2005).

[8] F. Glas. Phys. Rev. B 74, 121302 (2006).

[9] F. Glas. J. Appl. Phys. 62, 3201 (1987).

[10] S.Yu. Karpov. MRS Internet J. Nitride Semicond. Res. 3, 16 (1998).

[11] V.G. Dubrovskii, A.A. Koryakin, N.V. Sibirev. Mater. Des. 132, 400 (2017).

[12] V.G. Dubrovskii, N.V. Sibirev. Cryst. Growth Des. 16, 2019 (2016).

[13] A.A. Koryakin, N.V. Sibirev, D.A. Zeze, V.G. Dubrovskii. J. Phys. Conf. Ser. 643, 012007 (2015).

[14] J. Johansson, M. Ghasemi. Cryst. Growth Des. 17, 1630 (2017).

[15] S. Christiansen, M. Albrecht, H.P. Strunk, H.J. Maier. Appl. Phys. Lett. 64, 3617 (1994).

[16] J.Y. Shen, C. Chatillon, I. Ansara, A. Watson, B. Rugg, T. Chart. Calphad 19, 215 (1995).

[17] Handbook Series on Semiconductor Parameters / Ed. M. Levinstein, S. Rumyantsev, M. Shur. World Scientific, Singapore (1996). V. 1.

[18] R.C. Cammarata. Progr. Surface Sci. 46, 1 (1994).

[19] N. Li, T.Y. Tan, U. Gösele. Appl. Phys. A 90, 591 (2008).

Редактор Е.Ю. Флегонтова 